New composite shade will mask metal and stained dentition

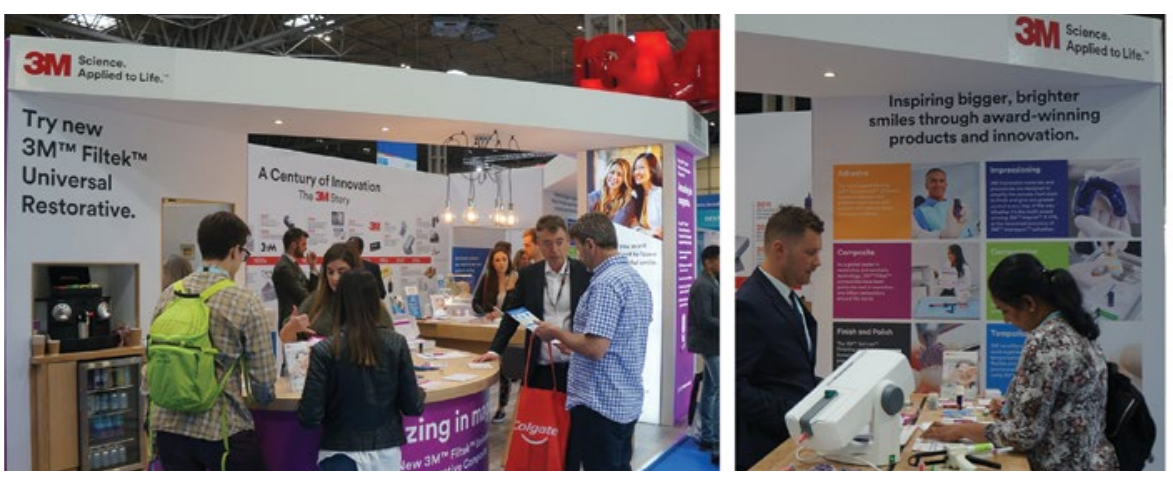

Delegates were excited to see a live demonstration of some of the new products from 3M Oral Care at the British Dental Conference and Dentistry Show this year. such as the new simplified shades of Filtek Universal Restorative plus the new Pink Opaquer shade from $3 \mathrm{M}$ which is a composite shade specially designed to mask metal and stained dentition. This was cured using the powerful Elipar DeepCure S by $3 \mathrm{M}$
Crowds gathered to see innovations

curing light, which delegates were delighted to see at work.

3M Oral Care also revealed the new, environmentally friendly packaging for the Filtek Universal Restorative.

If you missed seeing $3 \mathrm{M}$ Oral Care at the show, be sure to contact the team today to find out more. For more information, call 0800626578 or visit www.3M.co.uk/Dental

$3 \mathrm{M}$, Filtek and Elipar are trademarks of the 3M Company.

\section{All-time favourites}

As always, the Curaprox stand at the British Dental Conference and Dentistry Show proved hugely popular. Delegates were keen to sample innovative solutions such as the new Hydrosonic Ortho toothbrush, which has been designed to ensure a gentle but effective clean.

Delegates also had the opportunity to stock up on their all-time favourite products, including the CS 5460 manual toothbrush and CPS interdental brushes. The Black Is White and Be You range of toothpastes were also a big hit among delegates seeking to add a touch of personality to their oral healthcare regime.

Find out what other products Curaprox has to offer by contacting the friendly team today. For more information call 01480 862084, email info@curaprox.co.uk or visit www.curaprox.co.uk.

\title{
The home of Britain's top selling rubber interdental brush
}

Demonstrating its more than 235 years' experience in oral health, Wisdom Toothbrushes displayed its full product portfolio at the British Dental Conference and Dentistry Show.

This included the UK's No.1 selling rubber interdental brushes - Wisdom Clean Between Interdental Brushes - which are clinically proven to reduce gingival disease.

Delegates also discovered the Wisdom Pro-flex Wire Interdental Brushes, with a unique flexible hinge and curved profile that improves access to all areas of the mouth. The Wisdom Clean Between Easy Slide Tensioning Flossers with the unique patented Waveform Tension Control System were another hit, featuring taunt, silk-like tape to facilitate effortless and comfortable interdental cleaning.

Also, on the Friday, Wisdom partnered with Dr Barry Oulton who delivered a very popular lecture which offered some interesting insights into how dental professionals can improve patient compliance by reinforcing and embedding unconscious patterns of behaviour in order to encourage habit change in relation to interdental cleaning.

Find out more by contacting the team today.

To find out more, visit www.wisdomtoothbrushes.com or call 01440714800 .

\section{The world's first and only flossing toothbrush}

The Waterpik stand was as popular as ever at the British Dental Conference and Dentistry Show 2019.

Delegates had the chance to see all the latest products on offer including the newest Waterpik Water Flosser models plus the ever popular

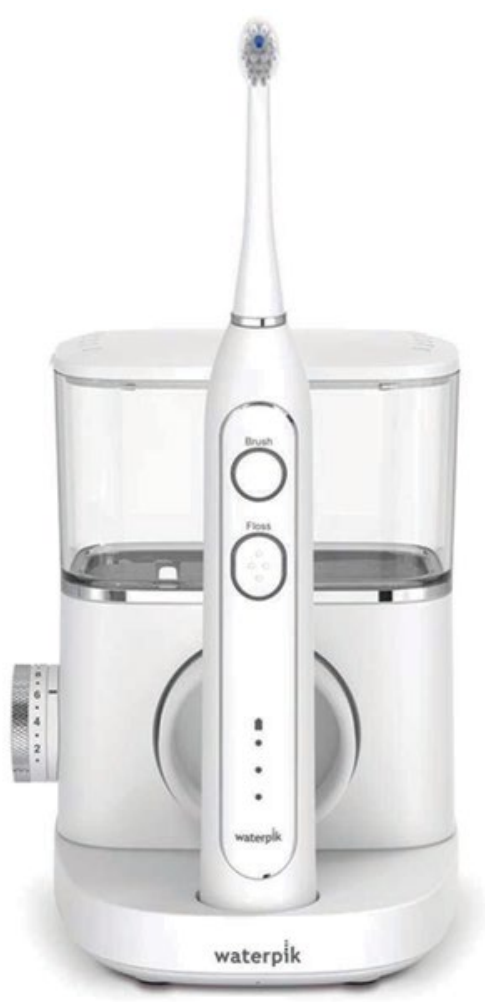

Whitening Water Flosser and Complete Care range. The star attraction was the Sonic-Fusion, the world's first and only flossing toothbrush; visitors were amazed by its oral healthcare benefits.

With products developed to improve and enhance your patient's home oral health routine, Waterpik is a resounding success.

If you missed the British Dental Conference and Dentistry Show but would like to learn more, contact the Waterpik team now.

For more information on Waterpik products visit www.waterpik.co.uk. Waterpik products are available from Amazon, Asda, Costco UK, Boots.com, Superdrug online and in stores across the UK and Ireland. 